\title{
Downregulation of secreted clusterin potentiates the lethality of sorafenib in hepatocellular carcinoma in association with the inhibition of ERK1/2 signals
}

\author{
JINGTAO ZHONG ${ }^{1}$, XIAOMING YU ${ }^{2}$, XIAOFENG DONG ${ }^{3}$, HONG LU $^{4}$, WUYUAN ZHOU $^{1}$, LEI LI $^{1}$, \\ ZHONGCHAO LI ${ }^{1}$, PENGFEI SUN ${ }^{1}$ and XUETAO SHI ${ }^{1}$ \\ ${ }^{1}$ Department of Hepatobiliary Surgery, Shandong Cancer Hospital Affiliated to Shandong University, \\ Shandong Academy of Medical Science, Jinan, Shandong 250117; \\ ${ }^{2}$ Department of Ophthalmology, Shandong Provincial Third Hospital, Jinan, Shandong 250031; \\ ${ }^{3}$ Department of Hepatobiliary Surgery, The People's Hospital of Guangxi Zhuang Autonomous Region, Nanning, \\ Guangxi 530021; ${ }^{4}$ Department of Radiology, Shandong Cancer Hospital Affiliated to Shandong University, \\ Jinan, Shandong 250117, P.R. China
}

Received July 14, 2017; Accepted February 2, 2018

DOI: $10.3892 /$ ijmm.2018.3463

\begin{abstract}
Secretory clusterin (sCLU) is overexpressed in cancer and is associated with resistance to chemotherapy in several types of cancer, including hepatocellular carcinoma (HCC). Sorafenib (SOR), a multikinase inhibitor of Raf/mitogen-activated protein kinase kinase/extracellular signal-regulated kinase (ERK) signaling and the receptor tyrosine kinase, is recognized as the standard therapeutic strategy for patients with advanced HCC. However, the role of sCLU in the resistance of HCC to SOR remains to be fully elucidated. In the present study, sCLU was silenced by CLU short hairpin (sh)RNA in Bel-7402 and SMMC-7721 cell lines, following which the cells were treated with SOR. Cell proliferation was determined using a CCK-8 assay. Apoptosis was quantified using flow cytometry. The production of sCLU, B-cell lymphoma 2 (Bcl-2), Bcl-2-associated X sprotein and phosphorylated (p)ERK1/2 was analyzed using western blot analysis. The results showed that sCLU was overexpressed in three HCC cell lines. The downregulation of sCLU by CLU shRNA synergistically increased SOR sensitivity in the Bel-7402 and SMMC-7721 cells, and potentiated SOR-induced cell apoptosis. In addition, silencing sCLU or combination with PD98059 decreased the SOR-induced activation of pERK1/2. These findings indicate a novel treatment strategy for HCC.
\end{abstract}

Correspondence to: Dr Jingtao Zhong or Professor Xuetao Shi, Department of Hepatobiliary Surgery, Shandong Cancer Hospital Affiliated to Shandong University, Shandong Academy of Medical Science, 440 Jiyan Road, Jinan, Shandong 250117, P.R. China E-mail: jingtao2005@126.com

E-mail: shixuetao9@126.com

Key words: hepatocellular carcinoma, clusterin, sorafenib, apoptosis, extracellular signal-regulated kinase

\section{Introduction}

Hepatocellular carcinoma (HCC) ranks as the sixth most common type of malignancy in the world and is the second leading cause of cancer-associated mortality (1). The development and progression of HCC is affected by various factors. Hepatitis virus infection (hepatitis B or C viruses), alcohol-related liver cirrhosis, and non-alcoholic steatohepatitis are the major risk factors for developing HCC (2). Surgery is the most effective treatment for HCC, although the curative resection ratio is low and the rate of recurrent metastasis remains high. Other treatment strategies depend on the tumor stage, and suitable candidates include radiofrequency ablation, transarterial chemoembolization and target therapy (3).

Molecule-targeted therapy, which aims to investigate carcinogenic mechanisms and molecular biology provides a novel approach in HCC treatment, particularly for patients with advanced HCC. Sorafenib (SOR), an oral multikinase inhibitor, was the first approved by the Food and Drug Administration for the treatment of 'unresectable' HCC, and has been considered as a novel molecular-targeted therapy for HCC as it prolongs survival rates by 2-3 months in cases of advanced and inoperable HCC $(4,5)$. SOR can inhibit tumor angiogenesis and growth by suppressing the RAS/RAF/mitogen-activated protein kinase (MAPK) signaling pathway and other extracellular receptor tyrosine kinases, including vascular endothelial growth factor receptor (6). However, clinical results have been disappointing; they have demonstrated that the prognosis of patients with HCC treated with SOR remained less than satisfactory (7). Therefore, it is urgent and necessary to identify the molecular mechanisms underlying hepatocarcinogenesis, and investigate novel therapeutic approaches to combat HCC.

Clusterin (CLU), a conserved heterodimeric disulfidelinked glycoprotein, is widely distributed in tissues and body fluids, and is crucial in tissue remodeling, reproduction, lipid transport, complement regulation and apoptosis (8-10). CLU appears to have two main isoforms, which result from 
alternative splicing. The secreted form of the CLU protein (sCLU) starts as a precursor peptide of $\sim 60 \mathrm{kDa}$, which represents the predominant translation product of the CLU gene. Nuclear clusterin is translated from an alternatively spliced CLU transcript, which bypasses the endoplasmic reticulum signal peptide $(11,12)$. sCLU has been recognized as an important contributor to chemoresistance against anticancer agents $(13,14)$. Our previous studies also demonstrated that sCLU contributes to oxaliplatin-resistance by activating the Akt pathway in HCC (15) and that downregulating sCLU enhance the sensitivity of HCC cells to gemcitabine via activating the intrinsic apoptotic pathway (16). However, the effect of sCLU on SOR in the treatment of HCC remains to be fully elucidated. Therefore, the present study tested the hypothesis that inhibiting sCLU enhances the sensitivity of HCC cells to SOR, and aimed to investigate the role and potential downstream pathways of sCLU in SOR-induced cytotoxicity in human HCC cells.

\section{Materials and methods}

Cell culture. The human HepG2, Bel-7402 and Bel-7404 HCC cell lines were obtained from the American Type Culture Collection (Rockville, MD, USA). The SMMC-7721 and Huh-7 cell lines were purchased from the Type Culture Collection Cell Bank, Chinese Academy of Science (Shanghai, China). The cells were cultured in RPMI-1640 medium (Gibco; Thermo Fisher Scientific, Inc., Waltham, MA, USA)/Dulbecco's modified Eagle's medium (DMEM; HyClone; GE Healthcare Life Sciences, Logan, UT, USA) containing $10 \%$ fetal bovine serum (FBS; Gibco; Thermo Fisher Scientific, Inc.), $100 \mathrm{U} / \mathrm{ml}$ penicillin and $100 \mu \mathrm{g} / \mathrm{ml}$ streptomycin (Gibco; Thermo Fisher Scientific, Inc.) at $37^{\circ} \mathrm{C}$ in $95 \%$ air and $5 \% \mathrm{CO}_{2}$.

Reagents and antibodies. SOR tosylate was purchased from Bayer AG (Berlin, Germany). The SOR was dissolved in 100\% dimethyl sulfoxide (DMSO) to produce a stock solution and was diluted with RPMI-1640/DMEM to the desired concentrations with a final DMSO concentration of $0.1 \%$ for the in vitro assays. PD98059, a specific inhibitor of ERK kinase, was obtained from Sigma-Aldrich; Merck Millipore (Darmstadt, Germany). Primary antibodies targeting B-cell lymphoma 2 (Bcl-2; cat. no. 2872) and Bcl-2-associated X protein (Bax; cat. no. 2772) were obtained from Cell Signaling Technology, Inc. (Danvers, MA, USA) and anti-GAPDH antibody (cat. no. ab37168) was obtained from Abcam (Cambridge, UK). Antibodies targeting sCLU (cat. no. sc-5289) and anti-ERK1/2 (Phospho-Thr202/Tyr204) (cat. no. sc-136521) antibodies were purchased from Santa Cruz Biotechnology, Inc. (Dallas, TX, USA).

Cell viability, apoptosis and western blot analysis. These methods were as previously described $(17,18)$. Briefly, cell viability assays were performed using the Cell Counting Kit-8 (CCK-8; Dojindo Molecular Technologies, Inc., Kumamoto, Japan). Cells (5x10\%/well) were seeded onto 96-well plates and incubated in DMEM (HyClone; GE Healthcare Life Sciences, Logan, UT, USA) supplemented with 10\% FBS (Gibco; Thermo Fisher Scientific, Inc.), $100 \mathrm{U} / \mathrm{ml}$ penicillin and $100 \mu \mathrm{g} / \mathrm{ml}$ streptomycin (Gibco; Thermo Fisher Scientific, Inc.) at $37^{\circ} \mathrm{C}$ for $24 \mathrm{~h}$. Following adaptation, cells were treated with different concentrations of $\operatorname{SOR}(0,2.5,5,7.5,10$ and $12.5 \mu \mathrm{M})$ for $48 \mathrm{~h}$. Finally, cells were incubated with DMEM (HyClone; GE Healthcare Life Sciences, Logan, UT, USA) supplemented with 10\% FBS (Gibco; Thermo Fisher Scientific,Inc.), $100 \mathrm{U} / \mathrm{ml}$ penicillin and $100 \mu \mathrm{g} / \mathrm{ml}$ streptomycin (Gibco; Thermo Fisher Scientific, Inc.) containing $10 \mu \mathrm{l}$ CCK- 8 solution at $37^{\circ} \mathrm{C}$ for $4 \mathrm{~h}$, and the optical density (OD) was measured at $450 \mathrm{~nm}$ using a Spectra Max 190 (Molecular Devices, LLC, Sunnyvale, CA, USA). For the apoptotic assay, the cells were incubated with $5 \mu \mathrm{l}$ of Annexin $\mathrm{V}$ and $5 \mu \mathrm{l}$ of propidium iodide for $15 \mathrm{~min}$ at room temperature in the dark, according to the manufacturer's protocol (BD Biosciences, San Jose, CA, USA), and were then subjected to flow cytometry to measure the apoptotic rate (\%). For the western blot analysis, cells were treated with either SOR $(0,2.5,5,7.5$ and $10 \mu \mathrm{M})$ alone, PD98059 $(5 \mu \mathrm{M})$ alone, or in combination (10 $\mu \mathrm{M}$ SOR and $5 \mu \mathrm{M}$ PD98059) for 3-24 h, and then lysed in cold radioimmunoprecipitation assay lysis buffer with $1 \mathrm{nM}$ phenylmethylsufonyl fluoride, followed by centrifugation at $12,000 \mathrm{xg}$ for $10 \mathrm{~min}$ at $4^{\circ} \mathrm{C}$. The protein concentrations in cell extracts were determined using a bicinchoninic acid assay (Bio-Rad Laboratories, Inc., Hercules, CA, USA). Total proteins (20-40 $\mu \mathrm{g})$ were separated using SDS-PAGE (10-12\% gel) and electrotransferred to polyvinylidene fluoride membranes (EMD Millipore, Billerica, MA, USA). Membranes were blocked using 5\% skimmed milk in Tris-buffered saline (T5912, pH 7.4; Sigma-Aldrich; Merck Millipore) containing 0.05\% Tween 20 (P1379; Sigma-Aldrich; Merck Millipore) and were incubated with primary antibodies overnight at $4^{\circ} \mathrm{C}(1: 1,000$ for sCLU, Bcl-2, Bax and pERK1/2). Membranes were then incubated with horseradish peroxidase-conjugated secondary antibodies (cat. no. 7074/7076; 1:2,000; Cell Signaling Technology, Inc.) for $1 \mathrm{~h}$ at room temperature followed by visualization with enhanced chemiluminescence immunoblotting detection reagents (Merck Millipore). The levels of protein band intensities were quantified using ImageJ software (version 1.6.0_20; National Institutes of Health, Bethesda, MD, USA).

Knockdown of sCLU using short hairpin (sh)RNA. Four pMAGic7.1-based shRNA vectors (CLU1, CLU2, CLU3 and CLU4), a scrambled shRNA vector (Table I) and a full-length human CLU cDNA (NM_203339; GenBank, National Institutes of Health) were provided by Shanghai Sunbio Biotechnology Co., Ltd. (Shanghai, China) (15). Transfection of cells was performed using GenJet DNA in vitro transfection reagent (SignaGen, Rockville, MD, USA).

Statistical analysis. Data are presented as the mean \pm standard deviation and were analyzed using one-way analysis of variance followed by Dunnett's test using SPSS software (version 17.0; SPSS, Inc., Chicago, IL, USA). P<0.05 was considered to indicate a statistically significant difference.

\section{Results}

HCC cells express sCLU and respond to SOR treatment. The present study first examined the protein expression of sCLU using western blot analysis. As shown in Fig. 1A and B, the HCC cell lines expressed different protein levels of sCLU, 
Table I. Sequences of oligonucleotides used as CLU RNAi primers.

\begin{tabular}{|c|c|c|c|}
\hline Construct & Position & Sequence & Target sequence \\
\hline CLU1 & $\begin{array}{l}\text { Upstream } \\
\text { Downstream }\end{array}$ & $\begin{array}{l}\text { 5'-CCGGGCTCCAGGAAATGTCCAATttcaagagaAT } \\
\text { TGGACATTTCCTGGAGCTTTTTTg-3' } \\
\text { 5'-AATTCAAAAAAGCTCCAGGAAATGTCCAAT } \\
\text { tctcttgaaATTGGACATTTCCTGGAGC-3' }\end{array}$ & GCTCCAGGAAATGTCCAAT \\
\hline CLU2 & $\begin{array}{l}\text { Upstream } \\
\text { Downstream }\end{array}$ & $\begin{array}{l}\text { 5'-CCGGGGTTGACCAGGAAATACAAttcaagaga } \\
\text { TTGTATTTCCTGGTCAACCTTTTTTg-3' } \\
\text { 5'-AATTCAAAAAAGGTTGACCAGGAAATACAA } \\
\text { tctcttgaaTTGTATTTCCTGGTCAACC-3' }\end{array}$ & GGTTGACCAGGAAATACAA \\
\hline CLU3 & $\begin{array}{l}\text { Upstream } \\
\text { Downstream }\end{array}$ & $\begin{array}{l}\text { 5'-CCGGGGGATATGATGACAAGGTTctcaagagaAA } \\
\text { CCTTGTCATCATATCCC TTTTTTg-3' } \\
\text { 5'-AATTCAAAAAAGGGATATGATGACAAGGTTtc } \\
\text { tcttgagAACCTTGTCATCATATCCC-3' }\end{array}$ & GGGATATGATGACAAGGTT \\
\hline CLU4 & $\begin{array}{l}\text { Upstream } \\
\text { Downstream }\end{array}$ & $\begin{array}{l}\text { 5'-CCGGCAGGGAAGTAAGTACGTCAATctcgagAT } \\
\text { TGACGTACTTACTTCCCTGTTTTTTg-3' } \\
\text { 5'-AATTCAAAAAACAGGGAAGTAAGTACGTCA } \\
\text { ATctcgagATTGACGTACTTACTTCCCTG-3' }\end{array}$ & CAGGGAAGTAAGTACGTCAAT \\
\hline Scramble & $\begin{array}{l}\text { Upstream } \\
\text { Downstream }\end{array}$ & $\begin{array}{l}\text { 5'-CCGGTTCTCCGAACGTGTCACGTttcaagagaTT } \\
\text { CTCCGAACGTGTCACGTTTTTTTg-3' } \\
\text { 5'-AATTCAAAAAATTCTCCGAACGTGTCACGTtc } \\
\text { tcttgaaTTCTCCGAACGTGTCACGT-3' }\end{array}$ & TTCTCCGAACGTGTCACGT \\
\hline
\end{tabular}

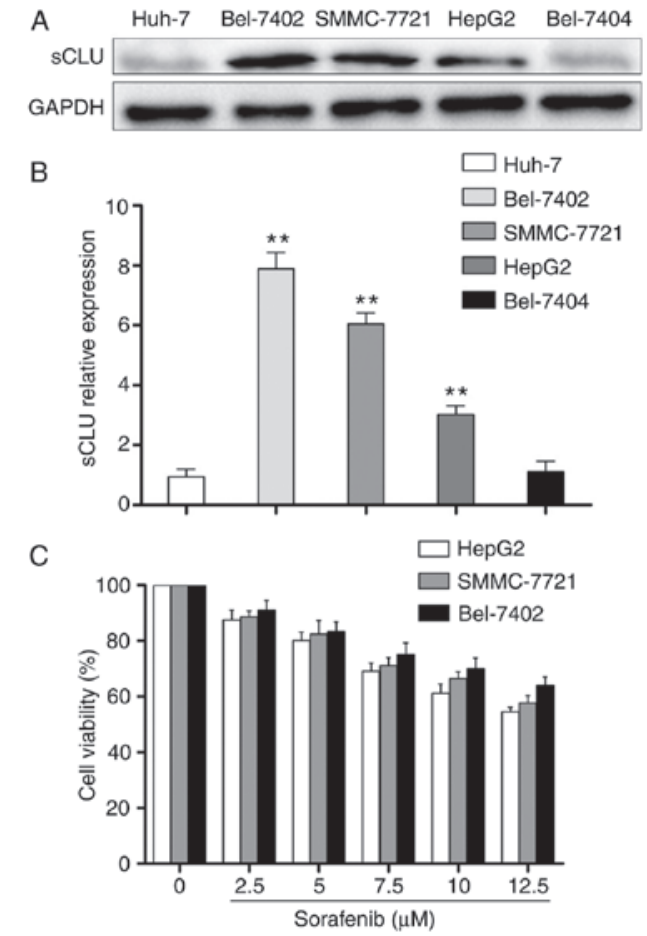

Figure 1. Expression of sCLU in HCC cells and SOR-induced reduction of cell viability in vitro. (A) Expression of sCLU in Huh-7, Bel-7402, SMMC-7721, HepG2, and Bel-7404 HCC cell lines was detected using western blot analysis. Levels of GAPDH served as a loading control. (B) Density of each band was measured and normalized to that of GAPDH, respectively. ${ }^{* *} \mathrm{P}<0.01$, vs. Huh-7. (C) Three HCC cell lines were incubated with SOR at various concentrations $(0-12.5 \mu \mathrm{M})$ for $48 \mathrm{~h}$ and cell viability was then determined using a CCK-8 assay. Data are representative of three independent experiments and are expressed as the mean \pm standard deviation. HCC, hepatocellular carcinoma; sCLU, secretory clusterin; SOR, sorafenib. and the Bel-7402, SMMC-7721 and HepG2 cells exhibited high expression levels of sCLU. However, the Huh-7 and Bel-7404 cells exhibited weak expression of sCLU. Therefore, the Bel-7402, SMMC-7721 and HepG2 cells were selected for examining the inhibitory effects of SOR on the viability of cells. The cells were treated with different concentrations of SOR $(0-12.5 \mu \mathrm{M})$ for $48 \mathrm{~h}$, and the inhibition of cell proliferation rates was assessed using a CCK- 8 assay. The results showed that all three HCC cell lines exhibited resistance to SOR treatment at different levels, particularly in the Bel-7402 cells (Fig. 1C). These results suggested that intrinsic level of sCLU in HCC cells was correlated with sensitivity to SOR.

SOR treatment upregulates sCLU in HCC cells in vitro. To examine whether the upregulation observed in the expression of sCLU is associated with SOR-induced resistance, Bel-7402 and SMMC-7721 cells were exposed to $10 \mu \mathrm{M}$ of SOR for 3-24 h, or with concentrations of 2.5-10 $\mu \mathrm{M}$ for $24 \mathrm{~h}$. As shown in Fig. 2A-D, SOR significantly increased the expression of sCLU in a time- and concentration-dependent manner. These data supported the hypothesis that the expression of sCLU may be altered in response to SOR treatment.

Silencing of sCLU enhances SOR-induced cytotoxicity in HCC cells in vitro. Chemoresistance is one of the main factors limiting effective cancer therapy. In the present study, to investigate whether the suppression of sCLU enhanced SOR-induced cytotoxicity, the cell viability of HCC cells transfected with CLU shRNA was determined. In our previous study (15), four pMAGic7.1-based shRNA vectors (CLU1, CLU2, CLU3 and CLU4) were designed to knock down the expression of sCLU 

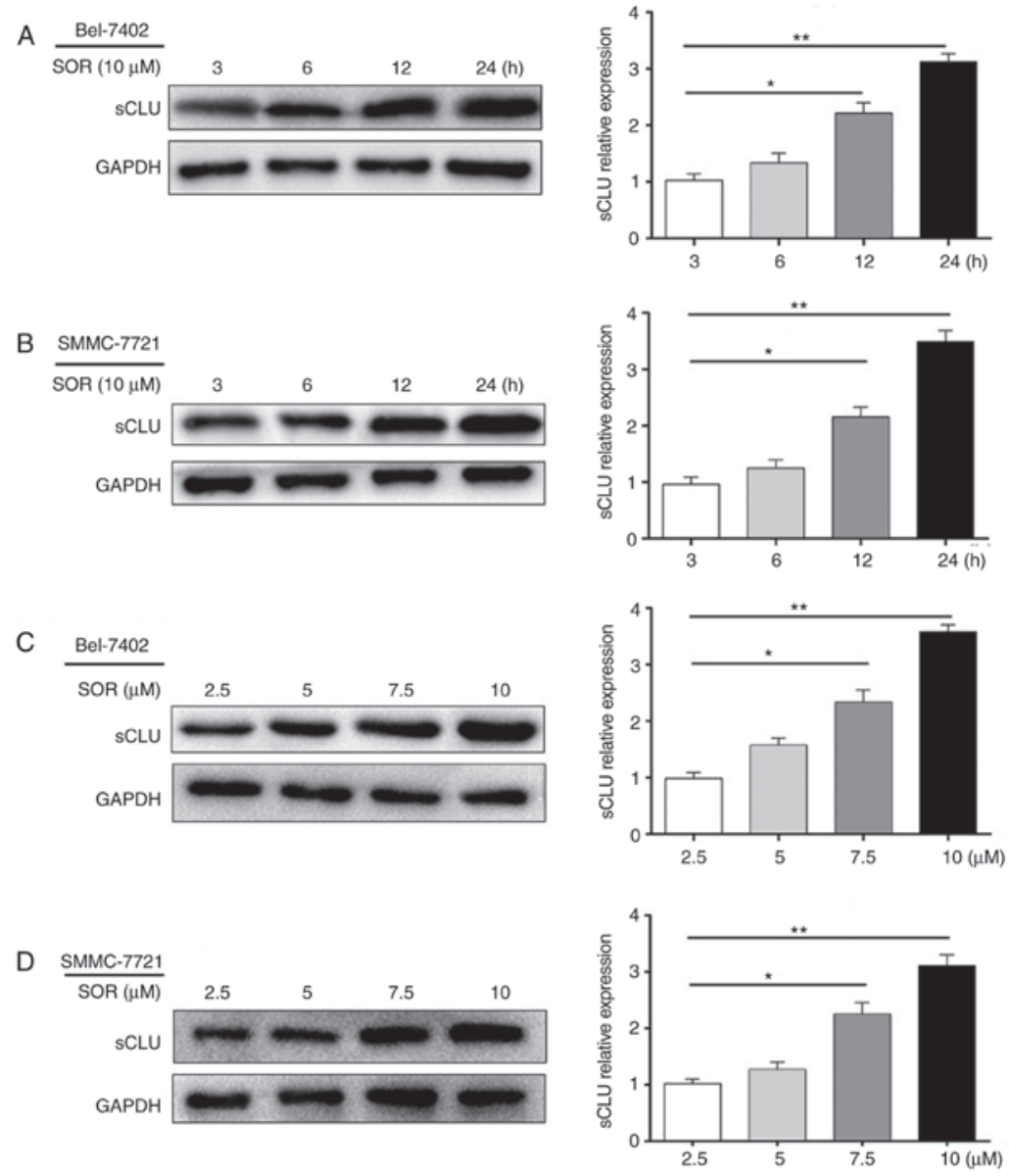

Figure 2. SOR treatment induces sCLU in a time- and concentration-dependent manner in hepatocellular carcinoma cells in vitro. Western blot analysis demonstrating the expression of sCLU in cell extracts of (A) Bel-7402 and (B) SMMC-7721 cells following treatment with $10 \mu \mathrm{M}$ SOR for 3-24 h. Levels of GAPDH served as a loading control. The density of each band was measured and normalized to that of GAPDH, respectively. ${ }^{*} \mathrm{P}<0.05$; ${ }^{* *} \mathrm{P}<0.01$. Western blot analysis of the expression of sCLU in cell extracts of (C) Bel-7402 and (D) SMMC-7721 cells following treatment with 2.5-10 $\mu$ M SOR for $24 \mathrm{~h}$. Levels of GAPDH served as a loading control. The density of each band was measured and normalized to that of GAPDH, respectively. ${ }^{*} \mathrm{P}<0.05$; ${ }^{* *} \mathrm{P}<0.01$. The data presented are representative of three independent experiments. sCLU, secretory clusterin; SOR, sorafenib.

A

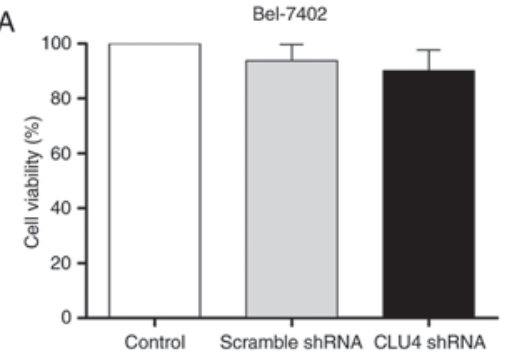

C

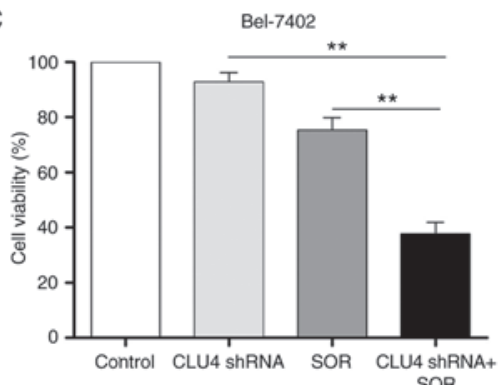

B

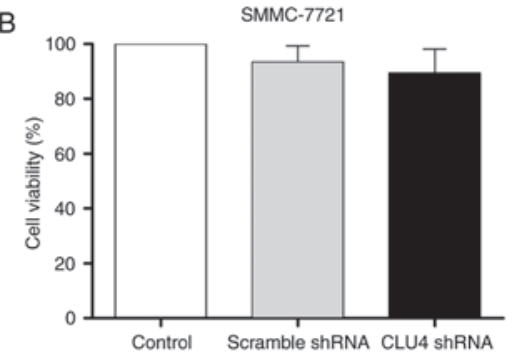

D

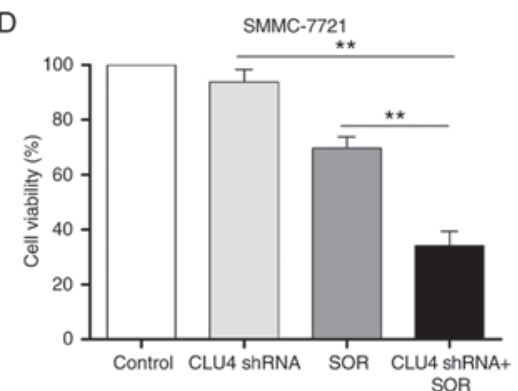

Figure 3. Silencing of sCLU enhances SOR-induced cytotoxicity in HCC cells in vitro. (A) Bel-7402 or (B) SMMC-7721 cells only (control) or transfected with scramble shRNA or the CLU4 shRNA vector, were cultured for $48 \mathrm{~h}$, and cell viability was measured using a CCK-8 assay. (C) Bel-7402 and (D) SMMC-7721 cells only or transfected with the CLU4 shRNA vector were cultured in medium with $10 \mu \mathrm{M}$ SOR for $48 \mathrm{~h}$, and cell viability was measured using a CCK-8 assay. ${ }^{* *} \mathrm{P}<0.01$. Data are representative of three independent experiments and are expressed as the mean \pm standard deviation. HCC, hepatocellular carcinoma; sCLU, secretory clusterin; SOR, sorafenib; shRNA, short hairpin RNA. 

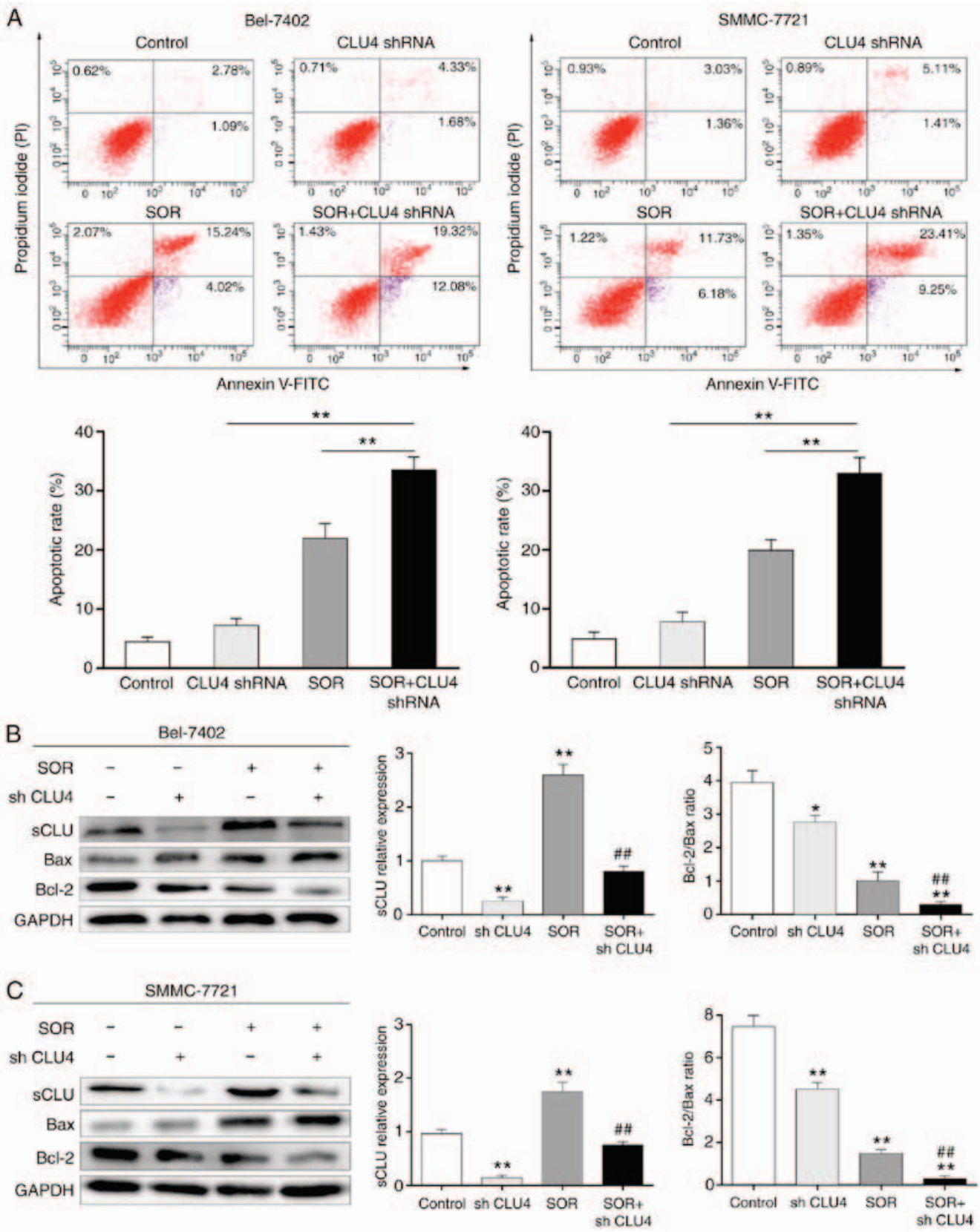

Figure 4. Combination effects of SOR and sCLU-knockdown on the apoptosis of hepatocellular carcinoma cells in vitro. (A) Bel-7402 and SMMC-7721 cells only (control), and the cells transfected with the CLU4 shRNA vector were incubated in the absence or presence of SOR (10 $\mu \mathrm{M})$ for $24 \mathrm{~h}$, and then cell apoptosis was determined using flow cytometry with Annexin V-FITC and propidium iodide. Quantitative analysis of the total apoptosis (early and late) population is presented. Data are presented as the mean \pm standard deviation of three independent experiments. ${ }^{* *} \mathrm{P}<0.01$. (B) Bel-7402 and (C) SMMC-7721 cells alone or the cells transfected with CLU4 shRNA vectors were incubated in the absence or presence of $10 \mu \mathrm{M}$ SOR for $24 \mathrm{~h}$. Cell lysates were harvested and analyzed using western blot analysis with specific antibodies against sCLU, Bax and Bcl-2. Levels of GAPDH served as a loading control. ${ }^{*} \mathrm{P}<0.05$ and ${ }^{* * *} \mathrm{P}<0.01$, vs. control; \#" $\mathrm{P}<0.01$, vs. SOR. The data shown are representative of three independent experiments. sCLU, secretory clusterin; SOR, sorafenib; shRNA, short hairpin RNA; Bcl-2, B-cell lymphoma 2; Bax, Bcl-2-associated X factor.

in HCC cell lines, and the results showed that CLU4 shRNA exhibited the most marked gene-silencing ability. Therefore, CLU4 shRNA was used in the present study. As shown in Fig. 3A and B, Bel-7402 and SMMC-7721 cell viabilities were only marginally altered by sCLU-knockdown. However, as shown in Fig. 3C and D, transfection with CLU4 shRNA notably reduced the viability of the Bel-7402 and SMMC-7721 cells treated with SOR. The SOR-induced expression of sCLU suggested the potential association between the expression of sCLU and apoptosis in Bel-7402 and SMMC-7721 cells. To clarify this, the role of sCLU in SOR-induced apoptosis of HCC cells was we examined. Transfection of the cells with CLU4 shRNA, which reduced the extent of sCLU, notably enhanced increase of cell apoptosis (Fig. 4A) and expression of Bax, and downregulated the expression of Bcl-2 (Fig. 4B and C) in the SOR $(10 \mu \mathrm{M})$-treated Bel-7402 and SMMC-7721 cells. These data suggested that the expression of sCLU may be correlated to the chemoresistance of human HCC cells to SOR.

SOR treatment induces the sCLU-dependent upregulation of pERK1/2 in human HCC cells in vitro. The activation of ERK1/2 leads to the phosphorylation of several intracellular 

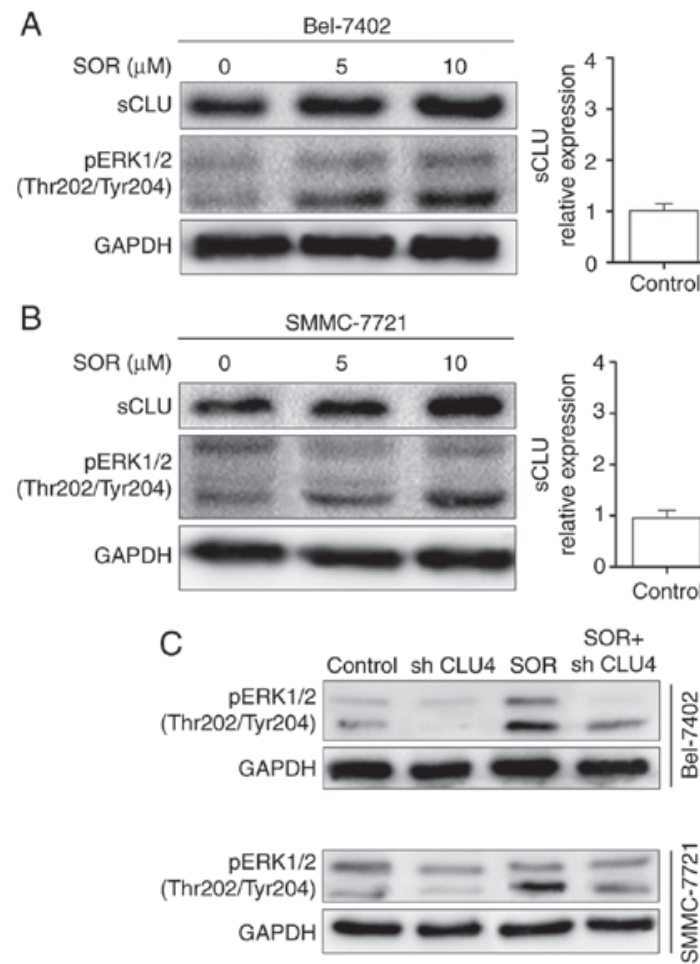
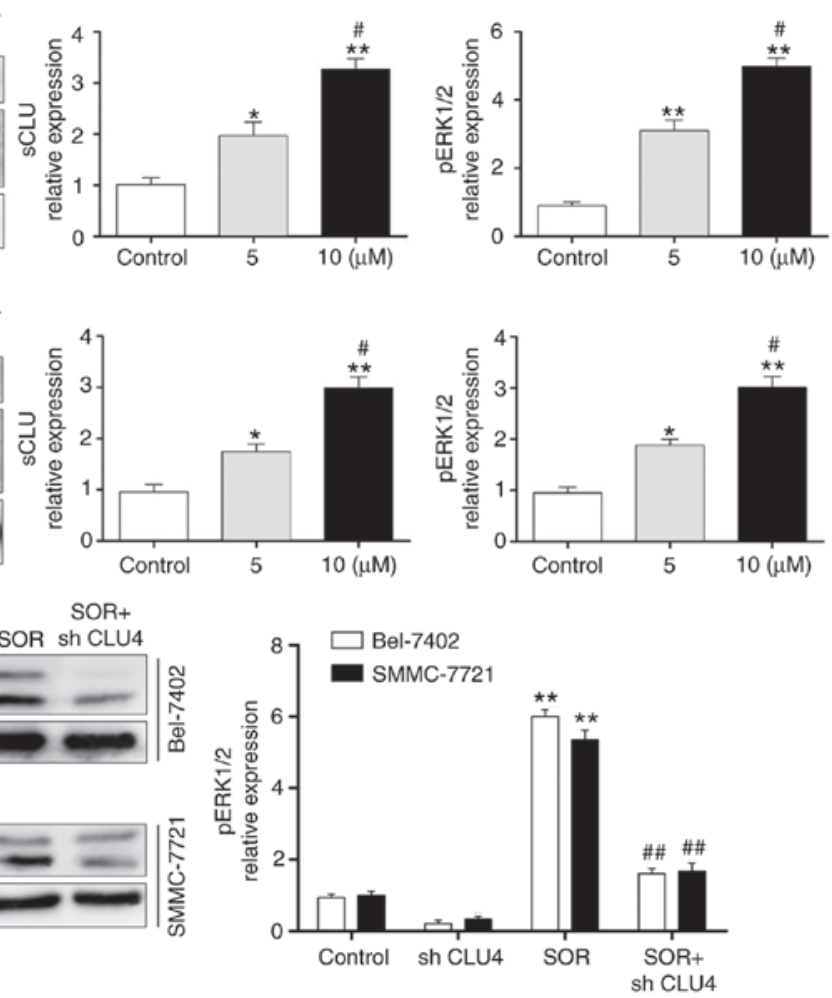

Figure 5. Regulation of the SOR-induced upregulation of pERK1/2 by sCLU. (A) Bel-7402 and (B) SMMC-7721 cells were treated with SOR (0-10 $\mu$ M) for $24 \mathrm{~h}$. Cell lysates were harvested and analyzed using western blot analysis with specific antibodies against sCLU and pERK1/2. Levels of GAPDH served as a loading control. ${ }^{*} \mathrm{P}<0.05$ and ${ }^{* *} \mathrm{P}<0.01$, vs. control. ${ }^{*} \mathrm{P}<0.05$, vs. SOR $(5 \mu \mathrm{M})$. (C) Bel-7402 and SMMC-7721 cells only, or the cells transfected with CLU4 shRNA vectors were incubated in the absence or presence of $10 \mu \mathrm{M}$ SOR for $24 \mathrm{~h}$. Cell lysates were harvested and analyzed using western blot analysis with specific antibodies against pERK1/2. Levels of GAPDH served as a loading control. "P<0.05 and ${ }^{* *} \mathrm{P}<0.01$, vs. control. ${ }^{\# \#} \mathrm{P}<0.01$, vs. SOR. The data shown are representative of three independent experiments. sCLU, secretory clusterin; SOR, sorafenib; shRNA, short hairpin RNA; pERK, phosphorylated extracellular signal-regulated kinase.

proteins, and is crucial in regulating cell growth, proliferation, invasion/migration, survival and motility (19-22). In the present study, the Bel-7402 and SMMC-7721 cells were treated with different concentrations of SOR $(0$ and $10 \mu \mathrm{M})$ for $24 \mathrm{~h}$. The protein expression levels were evaluated using western blot analysis. As shown in Fig. 5A and B, SOR significantly enhanced the expression of sCLU and pERK1/2 in a concentration-dependent manner. To investigate whether the protein expression of pERK1/2 was sCLU-dependent, the Bel-7402 and SMMC-7721 cells were transfected with CLU4 shRNA and then treated with SOR. As demonstrated in Fig. 5C, the combination treatment of SOR and CLU4 shRNA significantly decreased the activation of pERK1/2 in the two cell lines. These data suggested that SOR induced the sCLU-dependent activation of $\mathrm{pERK} 1 / 2$ in HCC cells.

Inactivated ERK1/2 sensitizes HCC cells to SOR treatment. The Bel-7402 and SMMC-7721 cells were treated with PD98059, an ERK-specific inhibitor, and then treated with different concentrations of SOR $(0$ and $10 \mu \mathrm{M})$ for 24 h. As shown in Fig. 6A and B, co-administration with PD98059 markedly abrogated the expression of pERK1/2 in the SOR-treated Bel-7402 and SMMC-7721 cells. To further examine whether the ERK1/2 signaling pathway protects HCC cells from SOR-induced cell death, PD98059 was used to inhibit pERK1/2. As expected, the Bel-7402 and SMMC-7721 cells were significantly more sensitive to SOR-induced apoptosis, compared with the cells treated with SOR in the absence of PD98059 (Fig. 6C). These results revealed that the inactivation of ERK1/2 is important in the potentiation of SOR lethality.

\section{Discussion}

Systemic chemotherapy is the only method capable of improving survival rates in patients with advanced HCC. However, the treatment of HCC using cytotoxic chemotherapy has reached a therapeutic bottleneck. Therefore, novel treatment modalities to treat HCC are urgently required. SOR, a multikinase inhibitor of Raf/MAPK kinase/ERK signaling and the receptor tyrosine kinase, has been recognized as the standard therapeutic strategy for patients with advanced HCC. However, the detailed mechanism of tumor lethality regulated by SOR and the precise mechanism of drug resistance have not been fully elucidated. In the present study, the results showed that SOR was capable of upregulating the expression of sCLU in human HCC cells. sCLU-knockdown using shRNA (CLU4 shRNA) or the ERK-inhibitor (PD98059) significantly potentiated the SOR-induced HCC cell apoptosis.

sCLU, also known as testosterone-repressed prostate message-2, apolipoprotein $\mathrm{J}$, or sulphated glycoprotein-2, is an ATP-independent and cytoprotective chaperone, which consists of two chains of $\sim 40 \mathrm{kDa}$ linked by five disulfide 

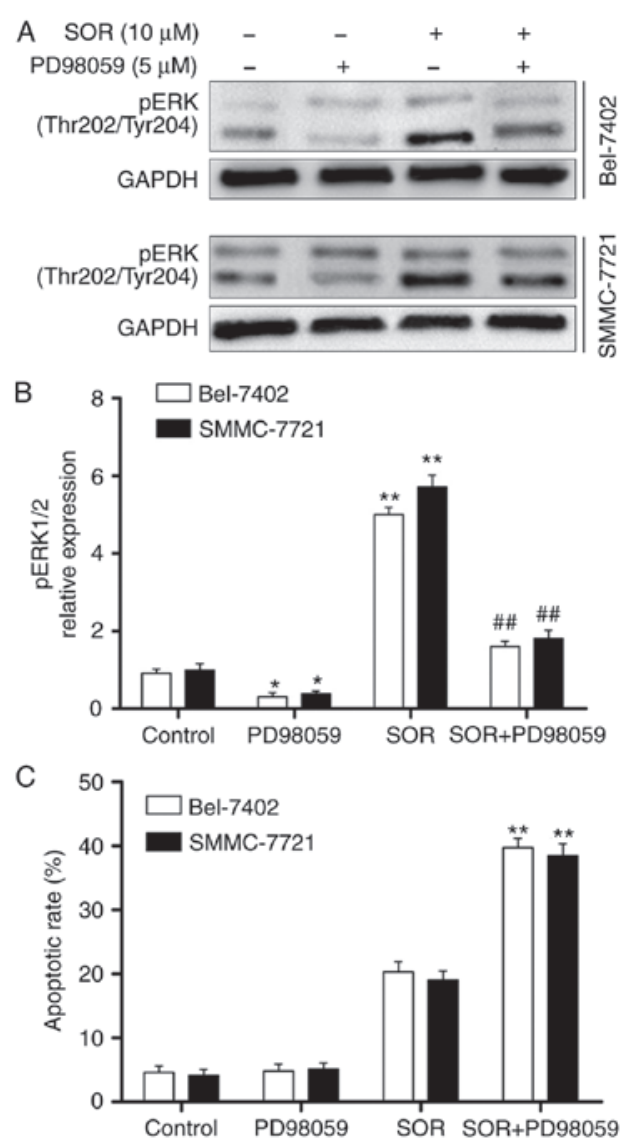

Figure 6. Inhibition of ERK1/2 sensitizes Bel-7402 and SMMC-7721 cells to SOR-induced apoptosis. (A) Cells were incubated in the presence of SOR $(10 \mu \mathrm{M})$ and PD98059 $(5 \mu \mathrm{M})$ individually or in combination. Cell lysates were harvested and analyzed using western blot analysis with specific antibodies against pERK1/2. Levels of GAPDH served as a loading control. (B) Quantification of results. ${ }^{*} \mathrm{P}<0.05$ and ${ }^{* *} \mathrm{P}<0.01$, vs. control ${ }^{\# \#} \mathrm{P}<0.01$, vs. SOR. The data shown are representative of three independent experiments. (C) Cells were incubated in the presence of SOR $(10 \mu \mathrm{M})$ and PD98059 $(5 \mu \mathrm{M})$ individually or in combination. Cells were stained with Annexin V-FITC and propidium iodide for analysis of apoptosis using FACS flow cytometry. Data are presented as the mean \pm standard deviation of three independents experiments. ${ }^{* *} \mathrm{P}<0.01$, vs. SOR. sCLU, secretory clusterin; SOR, sorafenib; shRNA, short hairpin RNA; pERK, phosphorylated extracellular signal-regulated kinase.

bonds $(23,24)$. sCLU is key in stabilizing the conformations of proteins during cellular stress, suppressing protein aggregation and precipitation, and protecting cells from numerous therapeutic factors (25-27). Accumulating evidence has also revealed that sCLU exerts inhibitory effects against cytotoxic chemotherapy in several types of cancer (28-31). The constitutive overexpression of sCLU has been recognized to confer drug-resistance in cancer therapy, whereas the downregulation of sCLU by RNA interference or chemical inhibition can enhance chemosensitivity (13,32-35). Our previous studies demonstrated that targeting sCLU enhances oxaliplatin or gemcitabine lethality in HCC via activating the Akt pathway or the intrinsic apoptotic pathway. However, the overexpression of sCLU significantly abrogates the inhibition of cell growth and the induction of cell apoptosis by oxaliplatin $(15,16)$. Chemoresistance remains one of the major factors leading to poor survival rates in patients with HCC. Identifying mechanisms of chemoresistance are important in the treatment of HCC. For the present study, it was hypothesized that sCLU is involved in the resistance of HCC to SOR. The results demonstrated that the treatment of Bel-7402 and SMMC-7721 cells with SOR induced the upregulation of sCLU. sCLU-knockdown using shRNA (CLU4 shRNA) significantly potentiated the cytotoxic and apoptotic effect of SOR in HCC cells. Taken together, thee results revealed the importance of sCLU in protecting cancer cells from SOR-induced apoptosis. Targeting sCLU in the two HCC cell lines examined distinctly increased their sensitivity to SOR.

The activation of ERK1/2 is important in regulating various cellular functions, ranging from proliferation to differentiation $(36,37)$. Several studies have reported that ERKs can also be activated in response to chemotherapeutic drugs $(38,39)$. In the present study, the data demonstrated that $\mathrm{pERK} 1 / 2$ was crucial in the resistance of Bel-7402 and SMMC-7721 cells to SOR. In addition, it was found that the inhibition of ERK1/2 by the ERK inhibitor (PD98059) significantly potentiated the chemotherapeutic potential of SOR in HCC cells in vitro. Several studies have demonstrated that sCLU is important in regulating the ERK1/2 signaling pathway $(40,41)$. Therefore, the present study investigated whether the downregulation of sCLU sensitizes Bel-7402 and SMMC-7721 cells to SOR chemotherapy via the ERK1/2 signaling pathway. The data revealed that the knockdown of sCLU by CLU4 shRNA sensitized HCC cells to SOR treatment through inhibiting the activation of pERK1/2. These results suggested that the downregulation of sCLU sensitized HCC cell lines to SOR via the pERK1/2-dependent signaling pathway.

In conclusion, SOR may affect the behavior of HCC cell lines through the upregulation of sCLU, which may be important in the effect of SOR. The downregulation of sCLU or exposure to the ERK inhibitor (PD98059) further potentiated SOR-induced HCC cell apoptosis. These findings indicate a potent strategy for the treatment against HCC.

\section{Acknowledgements}

The authors would like to thank Dr Edward C. Mignot, Shandong University (Shandong, China) for his linguistic advice.

\section{Competing interests}

The authors declare that they have no competing interests.

\section{References}

1. Siegel RL, Miller KD and Jemal A: Cancer statistics, 2017. CA Cancer J Clin 67: 7-30, 2017.

2. El-Serag HB: Hepatocellular carcinoma. N Engl J Med 365: 1118-1127, 2011.

3. Czaja AJ: Current management strategies for hepatocellular carcinoma. Minerva Gastroenterol Dietol 59: 143-159, 2013.

4. Llovet JM, Ricci S, Mazzaferro V, Hilgard P, Gane E, Blanc JF, de Oliveira AC, Santoro A, Raoul JL, Forner A, et al: Sorafenib in advanced hepatocellular carcinoma. N Engl J Med 359: 378-390, 2008.

5. Cheng AL, Kang YK, Chen Z, Tsao CJ, Qin S, Kim JS, Luo R, Feng J, Ye S, Yang TS, et al: Efficacy and safety of sorafenib in patients in the Asia-Pacific region with advanced hepatocellular carcinoma: A phase III randomised, double-blind, placebo-controlled trial. Lancet Oncol 10: 25-34, 2009. 
6. Wilhelm SM, Carter C, Tang L, Wilkie D, McNabola A, Rong H, Chen C, Zhang X, Vincent P, McHugh M, et al: BAY 43-9006 exhibits broad spectrum oral antitumor activity and targets the $\mathrm{RAF} / \mathrm{MEK} / \mathrm{ERK}$ pathway and receptor tyrosine kinases involved in tumor progression and angiogenesis. Cancer Res 64: 7099-7109, 2004.

7. Ogasawara S, Chiba T, Ooka Y, Kanogawa N, Motoyama T, Suzuki E, Tawada A, Kanai F, Yoshikawa M and Yokosuka O: Efficacy of sorafenib in intermediate-stage hepatocellular carcinoma patients refractory to transarterial chemoembolization. Oncology 87: 330-341, 2014

8. Yamamoto Y, Lin PJ, Beraldi E, Zhang F, Kawai Y, Leong J, Katsumi H, Fazli L, Fraser R, Cullis PR and Gleave M: siRNA lipid nanoparticle potently silence clusterin and delay progression when combined with androgen receptor cotargeting in enzalutamide resistant prostate cancer. Clin Cancer Res 21: 4845-4855, 2015.

9. Rubenstein M, Tsui P and Guinan P: Treatment of prostate and breast tumors employing mono- and bi-specific antisense oligonucleotides targeting apoptosis inhibitory proteins clusterin and bcl-2. Med Oncol 27: 592-599, 2010.

10. Rull A, Martinez-Bujidos M, Perez-Cuellar M, Pérez A, Ordóñez-Llanos J and Sánchez-Quesada JL: Increased concentration of clusterin/apolipoprotein $\mathrm{J}$ (apoJ) in hyperlipemic serum is paradoxically associated with decreased apoJ content in lipoproteins. Atherosclerosis 241: 463-470, 2015.

11. Trougakos IP, Djeu JY, Gonos ES and Boothman DA: Advances and challenges in basic and translational research on clusterin. Cancer Res 69: 403-406, 2009.

12. Trougakos IP, Lourda M, Antonelou MH, Kletsas D, Gorgoulis VG, Papassideri IS, Zou Y, Margaritis LH, Boothman DA and Gonos ES: Intracellular clusterin inhibits mitochondrial apoptosis by suppressing p53-activating stress signals and stabilizing the cytosolic Ku70-Bax protein complex. Clin Cancer Res 15: 48-59, 2009.

13. Zhang B, Zhang K, Liu Z, Hao F, Wang M, Li X, Yin Z and Liang H: Secreted clusterin gene silencing enhances chemosensitivity of a549 cells to cisplatin through AKT and ERK1/2 pathways in vitro. Cell Physiol Biochem 33: 1162-1175, 2014.

14. Koltai T: Clusterin: A key player in cancer chemoresistance and its inhibition. Onco Targets Ther 7: 447-456, 2014

15. Xiu P, Dong X, Dong X, Xu Z, Zhu H, Liu F, Wei Z, Zhai B, Kanwar JR, Jiang H, et al: Secretory clusterin contributes to oxaliplatin resistance by activating Akt pathway in hepatocellular carcinoma. Cancer Sci 104: 375-382, 2013

16. Xiu P, Xu Z, Liu F, Li Z, Li T, Zou F, Sun X and Li J: Downregulating sCLU enhances the sensitivity of hepatocellular carcinoma cells to gemcitabine by activating the intrinsic apoptosis pathway. Dig Dis Sci 59: 1798-1809, 2014.

17. Zhong J, Xiu P, Dong X, Wang F, Wei H, Wang X, Xu Z, Liu F, Li T, Wang Y and Li J: Meloxicam combined with sorafenib synergistically inhibits tumor growth of human hepatocellular carcinoma cells via ER stress-related apoptosis. Oncol Rep 34 2142-2150, 2015.

18. Dong X, Li R, Xiu P, Dong X, Xu Z, Zhai B, Liu F, Jiang H, Sun X, Li J and Qiao H: Meloxicam executes its antitumor effects against hepatocellular carcinoma in COX-2-dependent and -independent pathways. PLoS One 9: e92864, 2014.

19. Zhen Y, Zhang W, Liu C, He J, Lu Y, Guo R, Feng J, Zhang Y and Chen J: Exogenous hydrogen sulfide promotes C6 glioma cell growth through activation of the p38 MAPK/ERK1/2-COX-2 pathways. Oncol Rep 34: 2413-2422, 2015.

20. Li W, Liu Z, Zhao C and Zhai L: Binding of MMP-9-degraded fibronectin to $\beta 6$ integrin promotes invasion via the FAK-Src-related Erk1/2 and PI3K/Akt/Smad-1/5/8 pathways in breast cancer. Oncol Rep 34: 1345-1352, 2015.

21. Peng X, Zhou Y, Tian H, Yang G, Li C, Geng Y, Wu S and Wu W: Sulforaphane inhibits invasion by phosphorylating ERK1/2 to regulate E-cadherin and CD44v6 in human prostate cancer DU145 cells. Oncol Rep 34: 1565-1572, 2015

22. Peng X, Li F, Wang P, Jia S, Sun L and Huo H: Apelin-13 induces MCF-7 cell proliferation and invasion via phosphorylation of ERK1/2. Int J Mol Med 36: 733-738, 2015.

23. Shannan B, Seifert M, Leskov K, Willis J, Boothman D, Tilgen W and Reichrath J: Challenge and promise: Roles for clusterin in pathogenesis, progression and therapy of cancer. Cell Death Differ 13: 12-19, 2006.
24. Sowery RD, Hadaschik BA, So AI, Zoubeidi A, Fazli L, Hurtado-Coll A and Gleave ME: Clusterin knockdown using the antisense oligonucleotide OGX-011 re-sensitizes docetaxel-refractory prostate cancer PC-3 cells to chemotherapy. BJU Int 102: 389-397, 2008.

25. Muhammad LA and Saad F: The role of clusterin in prostate cancer: Treatment resistance and potential as a therapeutic target. Expert Rev Anticancer Ther 15: 1049-1061, 2015.

26. Song HB, Jun HO, Kim JH, Yu YS, Kim KW, Min BH and Kim JH: Anti-apoptotic effect of clusterin on cisplatin-induced cell death of retinoblastoma cells. Oncol Rep 30: 2713-2718, 2013.

27. Wang C, Jin G, Jin H, Wang N, Luo Q, Zhang Y, Gao D, Jiang $\mathrm{K}, \mathrm{Gu} \mathrm{D}$, Shen $\mathrm{Q}$, et al: Clusterin facilitates metastasis by EIF3I/Akt/MMP13 signaling in hepatocellular carcinoma. Oncotarget 6: 2903-2916, 2015.

28. Xiu P, Dong XF, Li XP and Li J: Clusterin: Review of research progress and looking ahead to direction in hepatocellular carcinoma. World J Gastroenterol 21: 8262-8270, 2015.

29. Niu ZH, Wang Y, Chun B, Li CX and Wu L: Secretory clusterin (sCLU) overexpression is associated with resistance to preoperative neoadjuvant chemotherapy in primary breast cancer. Eur Rev Med Pharmacol Sci 17: 1337-1344, 2013

30. Hassan MK, Watari H, Han Y, Mitamura T, Hosaka M, Wang L, Tanaka S and Sakuragi N: Clusterin is a potential molecular predictor for ovarian cancer patient's survival: Targeting clusterin improves response to paclitaxel. J Exp Clin Cancer Res 30: 113,2011

31. Zheng W, Sai W, Yao M, Gu H, Yao Y, Qian Q and Yao D: Silencing clusterin gene transcription on effects of multidrug resistance reversing of human hepatoma HepG2/ADM cells. Tumour Biol 36: 3995-4003, 2015.

32. Tang Y, Liu F, Zheng C, Sun S and Jiang Y: Knockdown of clusterin sensitizes pancreatic cancer cells to gemcitabine chemotherapy by ERK1/2 inactivation. J Exp Clin Cancer Res 31: 73, 2012.

33. Ma G, Cai H, Gao L, Wang M and Wang H: sCLU regulates cisplatin chemosensitivity of lung cancer cells in vivo. World J Surg Oncol 13: 80, 2015.

34. Zhang B, Liu ZM, Hao FG and Wang M: siRNA-directed clusterin silencing promotes cisplatin antitumor activity in human non-small cell lung cancer xenografts in immunodeficient mice. Eur Rev Med Pharmacol Sci 18: $1595-1601,2014$

35. Huang H, Wang L, Li M, Wang X and Zhang L: Secreted clusterin (sCLU) regulates cell proliferation and chemosensitivity to cisplatin by modulating ERK1/2 signals in human osteosarcoma cells. World J Surg Oncol 12: 255, 2014.

36. Dong F, Tian H, Yan S, Li L, Dong X, Wang F, Li J, Li C, Cao Z, Liu X and Liu J: Dihydroartemisinin inhibits endothelial cell proliferation through the suppression of the ERK signaling pathway. Int J Mol Med 35: 1381-1387, 2015.

37. Scherzad A, Steber M, Gehrke T, Rak K, Froelich K, Schendzielorz P, Hagen R, Kleinsasser N and Hackenberg S: Human mesenchymal stem cells enhance cancer cell proliferation via IL-6 secretion and activation of ERK1/2. Int J Oncol 47: 391-397, 2015.

38. Liu SQ, Xu CY, Qin MB, Tan L, Zhuge CF, Mao YB, Lai MY and Huang JA: Ginkgo biloba extract enhances chemotherapy sensitivity and reverses chemoresistance through suppression of the KSR1-mediated ERK1/2 pathway in gastric cancer cells. Oncol Rep 33: 2871-2882, 2015.

39. Wang LJ, Han SX, Bai E, Zhou X, Li M, Jing GH, Zhao J, Yang AG and Zhu Q: Dose-dependent effect of tamoxifen in tamoxifen-resistant breast cancer cells via stimulation by the ERK1/2 and AKT signaling pathways. Oncol Rep 29: 1563-1569, 2013.

40. Chou TY, Chen WC, Lee AC, Hung SM, Shih NY and Chen MY: Clusterin silencing in human lung adenocarcinoma cells induces a mesenchymal-to-epithelial transition through modulating the ERK/Slug pathway. Cell Signal 21: 704-711, 2009.

41. Shim YJ, Kang BH, Jeon HS, Park IS, Lee KU, Lee IK, Park GH, Lee KM, Schedin P and Min BH: Clusterin induces matrix metalloproteinase-9 expression via ERK1/2 and PI3K/Akt/NF- $\kappa \mathrm{B}$ pathways in monocytes/macrophages. J Leukoc Biol 90: 761-769, 2011. 